\title{
Effect of gefitinib, as a new stent coating material, on the cell proliferation and the expression of Caspase-3 and Bcl-2 in vascular smooth muscle cells
}

\author{
Ting Xiao ${ }^{1, \text { a }}$, Suyan Wang ${ }^{1}$, Jian Luo ${ }^{1}$, Zhixiong $\mathrm{Wu}^{1}$, Ou Zeng ${ }^{1}$, Fang $\mathrm{Li}^{1}$, \\ Jun Yang ${ }^{1, b}$ \\ ${ }^{1}$ Department of Cardiology, the First Affiliated Hospital of University of South China, Hengyang, \\ 421001, China \\ aemail:1459593600@qq.com, bemail:yangjunincn@163.com
}

Keywords: Coating material; Gefitinib; Cytotoxicity; Smooth muscle cells;

\begin{abstract}
To confirm the role and mechanism of gefitinib used as a new stent coating material on inhibition of smooth muscle cell proliferation, MTT assay was used to measure the cell proliferation and Western Blot was used to determine the expression of Caspase-3 and Bcl-2. Experiments showed that, compared with paclitaxel, gefitinib could effectively inhibit vascular smooth muscle cells (VSMCs) proliferation but had a less inhibitory effect on the proliferation of endothelial cells (ECs). What's more, Caspase3 was up-regulated while Bcl-2 was down-regulated more obviously in VSMCs after treated with gefitinib. This suggests it is possible for gefitinib to replace paclitaxel and other existing nonselective cytotoxic drugs used in the coating materials of the drug eluting stent to prevent restenosis after percutaneous coronary intervention (PCI).
\end{abstract}

\section{Introduction}

Now percutaneous coronary intervention (PCI) is the main method to treat the coronary heart disease. Drug-eluting stents have been widely used to reduce the occurrence of in-stent restenosis. However, Drug-eluting stents may cause endothelial damage and stent thrombosis, which is due to the non-selective cytotoxicity of the coating materials of the drug-eluting stent. To develop new stent coating materials, it is critical to selectively inhibit smooth muscle cells proliferation without affecting endothelial repair.

Several previous studies have shown that the migration of vascular smooth muscle cells (VSMCs) to the intima followed by excessive proliferation is the major mechanism of vascular restenosis after PCI [1]. The epidermal growth factor receptor (EGFR) mediated signaling pathway plays an important role in this process. Phenotypic modulation of VSMCs enables the expression of EGFR to increase by nearly ten times [2]. Therefore, EGFR is a critical intervention target in improving vascular remodeling. As a kind of important EGFR tyrosine kinase inhibitor (EGFR-TKI) [3], gefitinib competes for ATP binding site in intracellular region of EGFR tyrosine kinase pocket, and then holds back cell proliferation by blocking the phosphorylation of EGFR on the cell surface. While EGFR greatly increase on the surfaces of proliferated VSMCs undergoing phenotype remodeling, there is relatively low expression on the surfaces of endothelial cells (ECs). That means, gefitinib at a certain concentration may selectively inhibit the proliferation of VSMCs when exert a weak effect on the ECs. This study was designed to use MTT assay to measure the proliferation of VSMCs and ECs, and use Western Blot to determine the expression of Caspase-3 and Bcl-2 in VSMCs and ECs, aiming to provide experimental evidences for the application of gefitinib in stent coating materials to prevent restenosis after PCI.

\section{Materials and methods}

(1) Cells and Main Reagents. Rat vascular smooth muscle cells and endothelial cells were provided by the Experimental Animal Center, University of South China. Gefitinib and paclitaxel were purchased from AstraZeneca (England). Antibodies for caspase3 and Bcl-2 were purchased 
from Cell Signaling Technology (Beverly,MA). Goat anti-rat secondary antibody was purchased from Beijing tianlai Biotech.Cn.

(2) Cell Culture. Rat vascular smooth muscle cells and endothelial cells were respectively cultured in medium supplemented with $10 \% \mathrm{FBS}$ at $37^{\circ} \mathrm{C}$ in a humidified incubator with $5 \% \mathrm{CO}_{2}$. When they grew to $80 \%$ area of the Petri dish, the cells were subcultured 2 to 3 times after digestion with $0.25 \%$ trypsin.

(3) MTT Assay. About $10 \times 10^{4} / \mathrm{ml}$ cell suspension was made from logarithmic phase cells and $200 \mu \mathrm{l} /$ hole of it was added to 96 well plates, and then cultured in the incubator at $37^{\circ} \mathrm{C}$ with $5 \%$ $\mathrm{CO}_{2}$. Two hours later after cells adhered, the two drugs at the concentration of $10^{-2} \mu \mathrm{mol} \mathrm{L}{ }^{-1}$ were added respectively. 72 hours later, $20 \mu 1$ MTT was added into every hole, and then supplemented the hole with $150 \mu 1$ 10\% SDS 4 hours later. The absorbance at $490 \mathrm{~nm}$ was recorded using a 96-well microplate reader, and then calculated cell inhibition rate based on the absorbance value.

(4) Western Blot Analysis. Cells were lysed in protein lysis buffer and then were quantified. Each sample was subjected to 10\% SDS-PAGE and the separated proteins were transferred to PVDF membranes. The membranes were incubated with caspase 3 and Bcl-2 antibody respectively. Then primary antibodies were detected with a secondary antibody and finally the membranes were subjected to chemiluminescence detection assay.

(5) Statistical Analysis. SPSS19.0 was used for statistical analysis. Values were presented as mean \pm SD. The SNK method was used for comparisons .Difference were considered to be statistically significant if $P<0.05$.

\section{Results}

(1) Antiproliferative effects of gefitinib or paclitaxel on VSMCs and ECs

As indicated by markedly decreased absorbance values, both gefitinib and paclitaxel could obviously inhibit VSMCs proliferation at the concentration of $10^{-2} \mu \mathrm{mol} \mathrm{L} \mathrm{L}^{-1}$ after intervention for 72 hours. As shown in Figure 1, the inhibition rate of gefitinib and paclitaxel on VSMCs proliferation were $40.2 \%$ and $41.2 \%$ respectively. But gefitinib presented a less inhibitory effect on ECs proliferation than paclitaxel. That was the inhibition rate of gefitinib and paclitaxel on ECs proliferation were $30.6 \%$ vs $68.6 \%$.

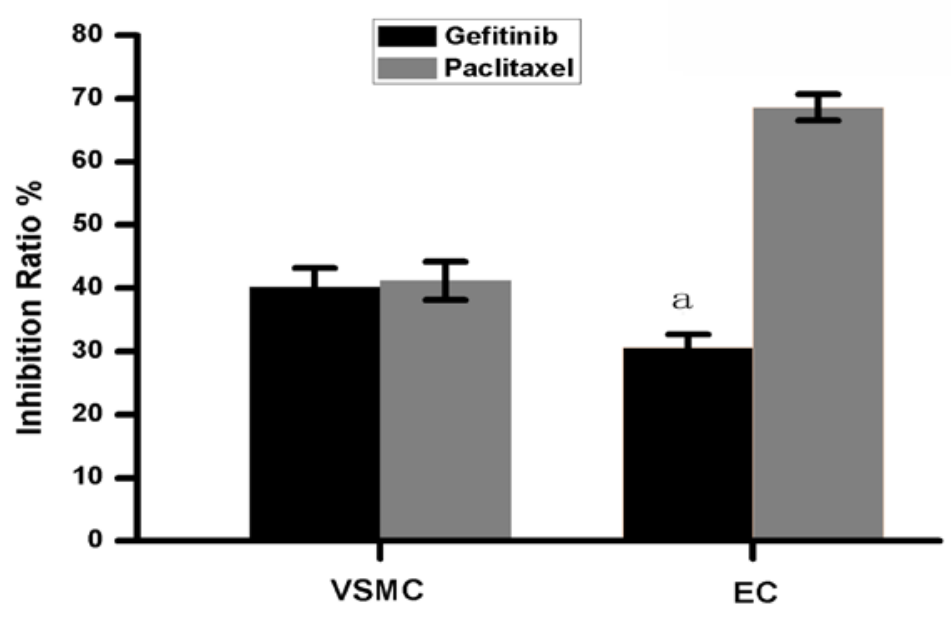

Fig.1 Inhibition rate of VSMCs and ECs proliferation after treated with gefitinib or paclitaxel ( ${ }^{\mathrm{a}} \mathrm{P}<0.05$ versus ECs treated with paclitaxel group).

(2) Effects of gefitinib or paclitaxel on caspase3 expression in VSMCs and ECs

As shown in Figure 2, apoptosis-related protein caspase3 was up-regulated in VSMCs and ECs after treated with gefitinib or paclitaxel. But compared with paclitaxel, gefitinib induced more evident expression of caspase3 in VSMCs. 


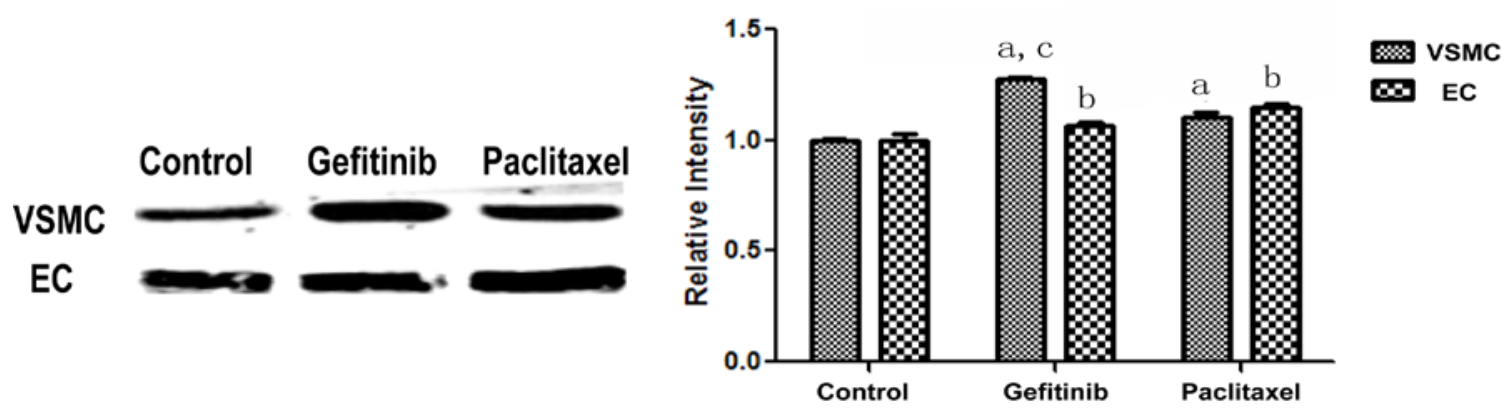

Fig.2 The expression of caspase3 in VSMCs and ECs after treated with gefitinib or paclitaxel ( ${ }^{\mathrm{a}} P<0.05$ versus control VSMCs group, ${ }^{\mathrm{b}} P<0.05$ versus control ECs group, ${ }^{\mathrm{c}} P<0.05$ versus VSMCs treated with paclitaxel group)

(3) Effects of gefitinib or paclitaxel on Bal-2 expression in VSMCs and ECs

As shown in Figure 3, anti-apoptotic protein Bcl-2 was down-regulated in VSMCs and ECs after treated with gefitinib or paclitaxel. But compared with paclitaxel, gefitinib induced much less expression of Bcl-2 in VSMCs.

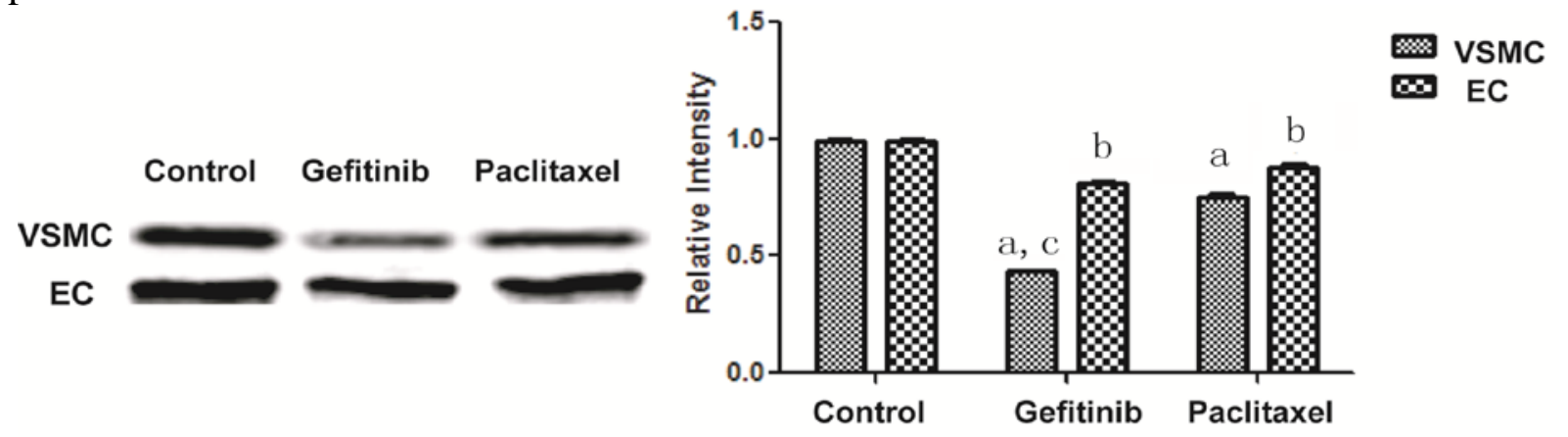

Fig.3 The expression of Bcl-2 in VSMCs and ECs after gefitinib or paclitaxel treatment ( ${ }^{\mathrm{a}} \mathrm{P}<0.05$ versus control VSMCs group, ${ }^{\mathrm{b}} \mathrm{P}<0.05$ versus control ECs group, ${ }^{\mathrm{c}} \mathrm{P}<0.05$ versus VSMCs treated with paclitaxel group)

\section{Discussion}

Paclitaxel and rapamycin have been proven to be effective in inhibiting VSMCs proliferation and now are widely used in stent coating materials to prevent restenosis after PCI. However, some scholars have found that paclitaxel not only significantly inhibit VSMCs proliferation and migration, but also inhibit the proliferation and migration of ECs, which will lead to the delay of stent endothelialization and increase the risk of in-stent thrombosis[4]. Hence, it is necessary to develop a drug that can inhibit VSMCs proliferation, but is less likely to hampering intimal restoration. The occurrence of vascular restenosis is mainly due to VSMCs' migration to the intima and their subsequent proliferations. A series of molecules and signal transduction systems involve in this process, including EGFR mediated signaling pathway. Gefitinib can combine with the intracellular region of EGFR to inhibit tyrosine kinase activity, and then inhibit EGFR phosphorylation and slow down the cell proliferation eventually. Researches from domestic and abroad have already shown that gefitinib can inhibit the proliferations of bladder smooth muscle cells and pulmonary arterial smooth muscle cells [5] [6]. The results of our research showed that compared with paclitaxel, gefitinib could also effectively inhibit VSMCs proliferation but had a less inhibitory effect on the proliferation of ECs. At the meantime, we observed Caspase3 was up-regulated and Bcl-2 was down-regulated in VSMCs and ECs after treated with gefitinib or paclitaxel. What's more, this change was most obvious in VSMCs group after gefitinib treatment, furthering supported the fact the cytotoxic effect of gefitinib on VSMCs is relatively selective. 
This study indicate that it is possible for gefitinib to replace paclitaxel and other existing nonselective cytotoxic drugs used in the coating materials of the drug eluting stent ,for it can reducing the incidence of in-stent restenosis as well as the risk of thrombosis.

\section{Acknowledgement}

In this paper, the research was sponsored by National Natural Science Foundation of China (81270181) and Hunan Provincial Natural Science Foundation of China (11JJ2046).

\section{References}

[1] Hoffmann R, Mintz GS, Dussaillant GR, et al. Patterns and mechanisms of in-stent restenosis. A serial intravascular ultrasound study[J]. Circulation,1996,94:1247-1254.

[2] Epsten SE, Siegall CB, Biro S, et al. Cytotoxic effects of a recombinant chimeric toxin on rapidly proliferating vascular smooth muscle cells [J]. Circulation, 1991, 84(2):778.

[3] Stefanini GG, Kalesan B, Serruys PW, et al. Long-term clinical outcomes of biodegradable polymer biolimus-eluting stents versus durable polymer sirolimus-eluting stents in patients with coronary artery disease (LEADERS): 4 year follow-up of a randomised non-inferiority trial[J]. Lancet 2011,378:1940-1948.

[4] Onuma Y, Garg S, Okamura T, et al. Ten-year follow-up of the IGAKI-TAMAI stent. A posthumous tribute to the scientific work of Dr. Hideo Tamai[J]. EuroIntervention, 2009,5 Suppl F:F109-111.

[5] Estrada CR, Adam RM, Eaton SH, Bagli DJ, et al. Inhibition of signaling abrogates smooth muscle proliferation resulting from sustained distention of the urinary bladder [J]. Laboratory Investigation, 2006, 86:1293-1302.

[6] Bhola KD, Teodora C, Aleksandra T, et al. Role of epidermal growth factor inhibition in experimental pulmonary hypertension[J].American Journal of Respiratory and Critical Care Medicine, 2010,181:158-167. 